\title{
Paciente inconsciente con hipernatremia adípsica: Un escenario de simulación a distancia con ayudas cognitivas para promover el razonamiento clínico en estudiantes de medicina
}

\author{
Unconscious patient with adipsic hypernatremia: \\ A remote simulation scenario with cognitive aids to \\ promote clinical reasoning in medical students \\ Paz Valenzuela-Bodenburg,* Juhi Datwani-Datwani,* \\ Joaquín Vrsalovic-Santibañez,* Soledad Armijo-Rivera,*,‡ \\ Cristian Labarca-Solar,* Edison Pablo Reyes, ${ }^{\S}$ Claudio Canals-Lambarri*
}

Palabras clave: Compromiso de conciencia, simulación remota, hipernatremia.

Keywords: Impaired consciousness, remote simulation, hypernatremia.

\footnotetext{
* Escuela de Medicina. ‡ Núcleo de Simulación Interdisciplinar. $\S$ Centro de Fisiología Celular e Integrativa.
}

Facultad de Medicina Clínica Alemana, Universidad del Desarrollo, Región Metropolitana. Chile.

Recibido: 13/08/2021 Aceptado: 07/11/2021

doi: $10.35366 / 103186$

\section{RESUMEN}

Introducción: El escenario de compromiso de conciencia para simulación remota permite a los estudiantes enfrentar una situación clínica desafiante para la práctica médica, usando un abordaje sistemático propuesto por expertos para orientar el diagnóstico y el tratamiento del paciente. Material y métodos: El escenario fue diseñado con base en un caso clínico real de hipernatremia adípsica, e implementado mediante una plataforma en línea. El desempeño de los estudiantes se evaluó con una pauta de acciones esperadas de razonamiento clínico, y se realizó debriefing apoyado por ayudas cognitivas diseñadas para el abordaje inicial y diagnóstico etiológico del compromiso de conciencia. Resultados: De las 19 acciones esperadas para el caso, los participantes abordaron espontáneamente 14 durante el escenario, dos aparecieron luego de indagaciones del instructor durante el debriefing y omitieron tres, $71 \%$ de las acciones esperadas en el escenario fueron realizadas por el médico a cargo, y el restante $30 \%$ se ejecutaron según las recomendaciones de otros miembros del equipo. Las ayudas cognitivas fueron bien valoradas por los estudiantes y guiaron la reflexión sobre la etiología del caso. Conclusiones: El diseño de este escenario y sus ayudas cognitivas permiten que los alumnos practiquen y reflexionen sobre el valor de un abordaje sistemático del compromiso de conciencia. Esta simulación puede efectuarse fácilmente de manera presencial o remota, favoreciendo el razonamiento clínico de los participantes.

\section{ABSTRACT}

Introduction: An impaired consciousness patient telesimulation scenario allows students to face a challenging clinical situation for medical practice, using a systematic approach proposed by experts to guide the diagnosis and treatment of the patient. Material and methods: The scenario was designed based on a real clinical case of adipsic hypernatremia, and implemented through an online platform. Student performance was evaluated with a guideline of expected clinical reasoning actions, and debriefing was conducted supported by cognitive aids designed for the initial approach and etiological diagnosis of the compromise of consciousness. Results: Of the 19 actions expected for the case, the active participants spontaneously approached 14 during the scenario, two appeared after instructor inquiries during debriefing, and three were omitted. $71 \%$ of the actions planned in the scenario were carried out by the doctor in charge of the case, $30 \%$ were carried out following the recommendations of other members of the team. The cognitive aids were well appreciated by the students and guided reflection on the etiology of the case. Conclusions: The design of this scenario and its cognitive aids allows learners to practice and reflect on the value of a systematic approach to impaired consciousness patients. This simulation can be easily performed in person or remotely, encouraging the clinical reasoning of the participants.

Citar como: Valenzuela-Bodenburg P, Datwani-Datwani J, Vrsalovic-Santibañez J, Armijo-Rivera S, Labarca-Solar C, Reyes EP, et al. Paciente inconsciente con hipernatremia adípsica: Un escenario de simulación a distancia con ayudas cognitivas para promover el razonamiento clínico en estudiantes de medicina. Rev Latinoam Simul Clin. 2021; 3 (3): 104-109. https://dx.doi. org/10.35366/103186 


\section{INTRODUCCIÓN}

$\mathrm{E}$ I compromiso de conciencia es un escenario clínico desafiante para los profesionales de la salud, dado principalmente por un contexto de urgencias sumado a un paciente que proporciona poca o nula información. Se estima que $1 \%$ de las consultas en servicio de urgencias corresponde a pacientes con alteraciones en el estado de conciencia. ${ }^{1}$ Un manejo para la estabilización inicial, la sospecha diagnóstica y el tratamiento de la causa sospechada son fundamentales para disminuir la morbimortalidad de estos pacientes.

Se han desarrollado escalas para evaluar el nivel de conciencia del paciente, la más conocida a nivel mundial es la escala de Glasgow, que evalúa tres parámetros clínicos principales: respuesta ocular, verbal y motora. ${ }^{2,3}$

Dentro del espectro de causas posibles, esta enfermedad puede ser producida por cualquier alteración que afecte los hemisferios cerebrales o estructuras subcorticales, ya que el estado de conciencia depende de dos estructuras, la corteza cerebral y el sistema reticular activador ascendente (SRAA). ${ }^{2,4}$ Por lo tanto, la etiología puede abarcar desde un trauma encefalocraneano o accidente cerebrovascular hasta alteraciones metabólicas.

Si bien existen algunas propuestas de abordaje sistemático del tema, ${ }^{5}$ el enfrentamiento de esta condición resulta desafiante en el proceso de enseñanza a estudiantes de pregrado médico. En un intento por combatir este problema, internistas expertos en el tema desarrollaron una forma de abordaje en cuatro pilares o grupos de patologías y una ayuda cognitiva visual para la óptima orientación de los estudiantes al enfrentar simulaciones de compromiso de conciencia.

En el caso del paciente de esta simulación, el compromiso de conciencia se produce por un mecanismo inusual, consistente en que la falta de ingesta de líquidos de este paciente no es censada correctamente por los osmorreceptores en el hipotálamo, determinando una disfunción de los mecanismos del control de la osmolaridad sérica, perpetuando la deshidratación por la incapacidad para sentir sed que presenta el paciente. La hipernatremia adípsica se describe poco en la literatura, y se asocia a lesiones hipotalámicas causadas por mecanismos diversos, ${ }^{6}$ trastornos psiquiátricos ${ }^{7} \mathrm{o}$ enfermedades autoinmunes. ${ }^{8}$

Un escenario de esta naturaleza promueve el razonamiento clínico, pues obliga a pensar más allá de las causas frecuentes, e impulsa el procesamiento analítico una vez constatado que el reconocimiento de patrones no permite resolver el caso con facilidad. 9,10

Un escenario cuya causa descansa en una alteración metabólica vinculada a una disfunción hipotalámica requiere de elementos de simulación que pueden ser trasladados de la simulación presencial a la transmisión remota por una plataforma de videoconferencias. Esta posibilidad constituye una oportunidad para mantener el desarrollo de la competencia de razonamiento clínico en contextos de distanciamiento físico como el que vivimos en los últimos años, y que ha forzado el desarrollo de innovaciones didácticas en diversas áreas. ${ }^{11,12}$

\section{PRESENTACIÓN DEL CASO}

Información del escenario: título: compromiso de conciencia por hipernatremia; nombre del paciente: Juan González; fecha de diseño: marzo de 2020. Apropiado para: estudiantes e internos de medicina, residentes de medicina y urgencia, médicos y enfermeras de urgencia y cuidado crítico.

Diseño del escenario: comienza con un paciente con compromiso cuantitativo de conciencia, acompañado de su hijo quien entrega los datos de la historia clínica y actúa como confederado. Historia de compromiso de conciencia de dos semanas de evolución, además de una historia de larga data de pérdida de memoria, asociada a agresividad y trastornos del lenguaje.

Objetivos: para el final de la simulación los estudiantes serán capaces de:

1. Realizar evaluación inicial del paciente.

2. Reconocer compromiso de conciencia, realizar un enfrentamiento con pasos ordenados y establecidos.

3. Solicitar exámenes de laboratorio e imágenes para identificar la etiología del compromiso de conciencia.

4. Realizar manejo correcto del compromiso de conciencia.

\section{Acciones críticas:}

1. Obtener la historia pertinente.

2. Realizar evaluación inicial y examen físico.

3. Identificación inicial del compromiso de conciencia.

4. Definir tipo de compromiso de conciencia. a. Compromiso difuso cortical.

b. Daño en formación reticular ascendente.

5. Planteamiento de cuatro dominios etiológicos. a. Estructural. 
b. Neurológico: vascular, síndrome convulsivo.

c. Infeccioso.

d. Tóxico metabólico.

6. Evaluación inicial de campos etiológicos del compromiso de conciencia (presencia o ausencia de signos meníngeos, antecedente o signos evidentes al examen físico de trauma, focalidad neurológica, causa metabólica).

7. Solicitar las pruebas diagnósticas y marcadores de gravedad (laboratorio y TC cerebral).

8. Evaluar los resultados.

9. Reconocer (verbalizar) la necesidad de manejo metabólico y respiratorio.

10. Hospitalizar y trasladar.

\section{Preparación del escenario:}

1. Software de simulación LLEAP Fundamentals (TM).

2. Plataforma de videoconferencia Zoom.

\section{Instrucciones al confederado:}

1. Entregue espontáneamente esta información: usted acompaña a su padre Don Juan de 87 años. Lo trae a urgencias porque está muy "raro".

2. Proporcione información adicional sólo cuando le hagan preguntas específicas.

Antecedentes de hipertensión arterial, daño hepático crónico, hipotiroidismo, enfermedad renal crónica y accidente cerebrovascular (ACV), isquémico hace seis años, secuelado. Usted vive con él y lo ha visto extraño las últimas dos semanas; está más somnoliento, lo cual ha aumentado en los últimos días y además responde poco a estímulos verbales. No ha tenido trauma, fiebre, convulsiones, tos o dificultad respiratoria, vómitos, consumo de fármacos nuevos o sustancias. Además presenta historia de tres años de evolución de pérdida de memoria asociada a agresividad y trastorno del lenguaje, y hace tres meses se encuentra postrado voluntariamente con disminución de interacción social y con familiares. Sus medicamentos son levotiroxina, atorvastatina y aspirina, no recuerda dosis. Ha notado que su ingesta de líquidos es escasa.

Laboratorios relevantes: electrolitos $\mathrm{Na} 164$ / $\mathrm{K} 5,5 / \mathrm{Cl} 122$; gasometría de sangre venosa (GSV): pH 7.34, pCO2 40, HCO3 21.1, AG 20.5; nitrógeno ureico en sangre (BUN) 89, creatinina 3.01; hematocrito $46 \%$, hemoglobina $15 \mathrm{~g} / \mathrm{dL}$; leucocitos
13,200 (70\% segmentado 19\% linfocitos); plaquetas 86,000; AST 25, ALT 40 total bilirrubina 0.92; Ca 10.3, P 5.4; orina normal, urocultivo tomado.

TC cerebral: secuelas isquémicas en el territorio superficial anterior de la arteria cerebral media (ACM) izquierda. Pequeñas secuelas isquémicas talámicas bilaterales. Microangiopatía de la sustancia blanca supratentorial.

\section{Preguntas para orientar el debriefing:}

1. ¿Tienes un enfoque predeterminado cada vez que te enfrentas a alguien con compromiso de conciencia?

2. ¿Por qué este cuadro es una urgencia que requiere tratamiento oportuno?

3. ¿Cuáles fueron los diagnósticos diferenciales que planteaste en este paciente?

4. ¿Cuál es el manejo de reanimación inicial de un paciente comprometido de conciencia?

5. ¿Cuáles son las complicaciones de la hipernatremia?, ¿cuáles son las complicaciones de un manejo inadecuado de la hipernatremia?

6. ¿En qué unidad tratarías al paciente?

\section{Implementación del escenario}

Se describen los resultados de la primera implementación en sala virtual para una audiencia de 32 estudiantes de cuarto año de medicina. El escenario remoto lo manejó inicialmente un estudiante que hizo de médico junto a una estudiante que hizo de enfermera. Ambos realizaron la mayor parte de la recabación de información (médico recaba información del familiar del paciente y enfermera del monitor). A los 10 minutos solicitaron apoyo a un segundo estudiante que actuó de médico y amplió las indagaciones mediante la solicitud de exámenes de laboratorio y recabación de información del familiar del paciente buscando causas tóxico metabólicas. Después, desde el chat, los observadores de la simulación complementaron con acciones de integración de información relacionadas (laboratorio y terapia). Se realizó un debriefing apoyado en ayuda cognitiva (Figura 1).

De las 19 acciones esperadas para el manejo ideal del caso, los participantes activos de la simulación abordaron espontáneamente 14 durante el escenario, dos las indagaron en respuesta a salvavidas introducidos en el escenario 
Enfrentamiento del compromiso de conciencia
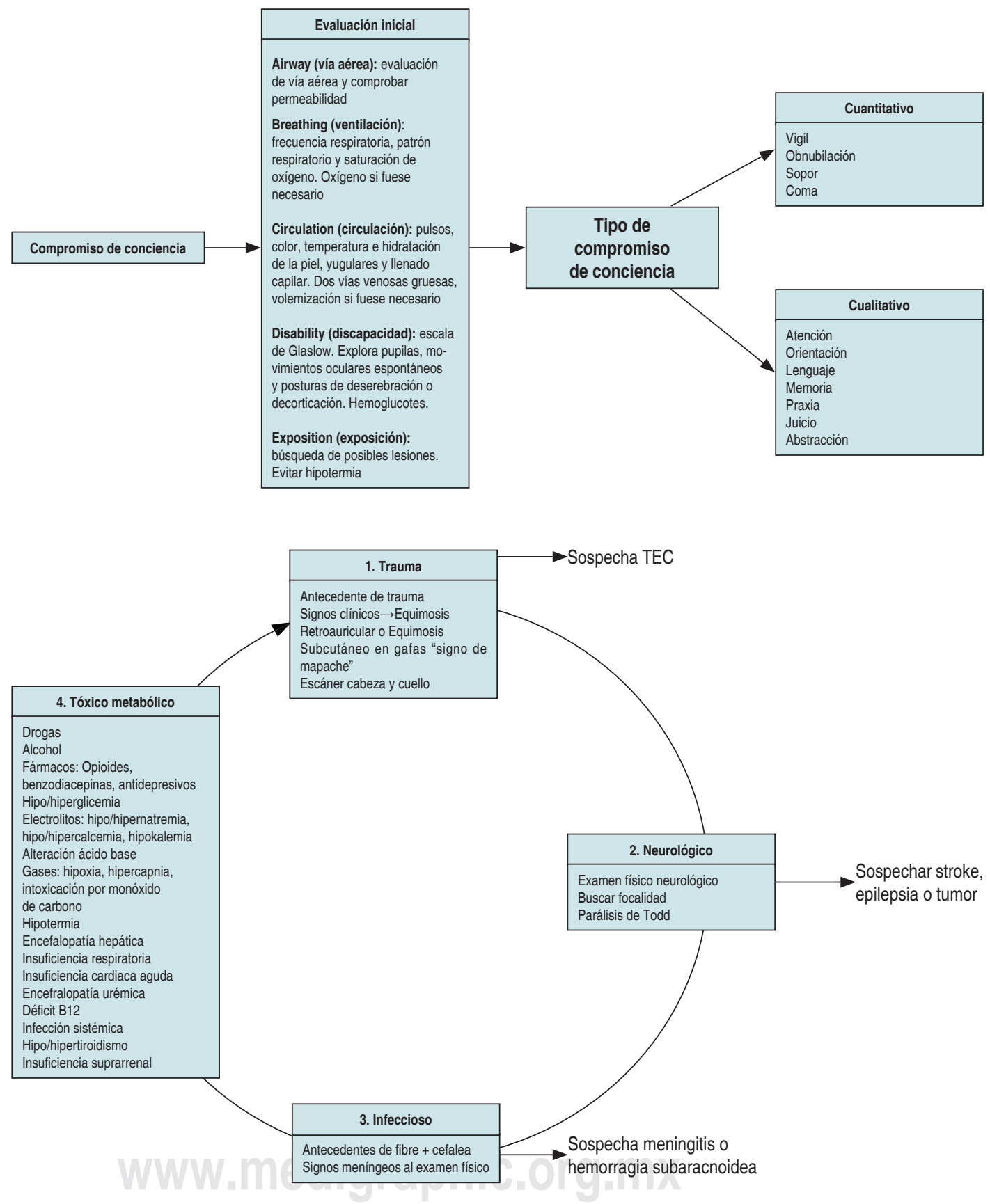

Figura 1: Ayuda cognitiva compromiso de conciencia.

Paciente inconsciente con hipernatremia adípsica: un escenario de simulación a distancia con ayudas cognitivas para promover el razonamiento clínico en estudiantes de medicina. 
por el instructor y omitieron tres, que más tarde se abordaron en el debriefing; $70 \%$ de las acciones esperadas en el escenario fueron realizadas por el médico a cargo del caso, y el restante $30 \%$ se ejecutó luego de las recomendaciones de otros miembros del equipo que atendieron la simulación.

Las acciones realizadas durante el escenario provinieron en mayor proporción del médico 1 (16/63) y la enfermera (14/63), quienes dirigieron buena parte de la recabación de información. Las acciones observadas en el escenario se clasificaron en categorías dentro del proceso de razonamiento clínico y de trabajo en equipo, observándose que hubo un alto número de acciones de recabación de información desde diversas fuentes dentro del escenario, y que para resolver el caso se hizo necesario desplegar acciones de trabajo en equipo, donde se compartió el modelo mental y se distribuyó la carga de trabajo (Tabla 1). La opinión de los alumnos sobre la utilidad de la simulación, el valor educativo del escenario y la ayuda cognitiva asociada fue positiva.

\section{DISCUSIÓN}

La implementación de la simulación remota de compromiso de conciencia en pacientes con hipernatremia, en estudiantes de cuarto año de medicina, demostró que para abordar un caso complejo en este nivel de formación es necesario el abordaje colaborativo de un equipo de estudiantes involucrados en la simulación de manera activa con apoyo de observadores participantes en el chat.

Esta simulación fue creada para dar a conocer a los estudiantes de medicina y a médicos en formación un manejo metódico y lógico para encontrar la causa del compromiso de conciencia en un paciente. A pesar de que la consulta al servicio de urgencias por compromiso de conciencia ocupa un lugar importante, no hallamos en la literatura un esquema de enfrentamiento establecido para que el personal de salud enfrente un caso como éste.

Un escenario de esta naturaleza puede presentarse de manera presencial utilizando simuladores de alta fidelidad, con pacientes y monitores simulados, y en este último caso es posible presentarlo de manera remota, guiando dicho escenario mediante un monitor simulado transmitido por una plataforma de videoconferencias. En todos los casos, las limitaciones de

\begin{tabular}{|c|c|}
\hline \multicolumn{2}{|c|}{$\begin{array}{l}\text { Tabla 1: Acciones observadas } \\
\text { en el escenario remoto. }\end{array}$} \\
\hline Categoría & \\
\hline \multicolumn{2}{|l|}{ Razonamiento clínico } \\
\hline $\begin{array}{l}\text { Recabación de información } \\
\text { desde el familiar del paciente }\end{array}$ & 14 \\
\hline $\begin{array}{l}\text { Recabación de información del } \\
\text { examen físico del paciente }\end{array}$ & 6 \\
\hline $\begin{array}{l}\text { Recabación de información } \\
\text { del monitor }\end{array}$ & 4 \\
\hline $\begin{array}{l}\text { Entrega de información } \\
\text { del entorno o contexto }\end{array}$ & 5 \\
\hline Integración de información & 9 \\
\hline Confirmación de información & 10 \\
\hline \multicolumn{2}{|l|}{ Trabajo en equipo } \\
\hline Distribuir la carga cognitiva & 6 \\
\hline Compartir modelo mental & 8 \\
\hline \multicolumn{2}{|l|}{ Comunicación hacia el paciente } \\
\hline Comunicación hacia el paciente & 1 \\
\hline
\end{tabular}

realismo de los simuladores obligan a que el examen neurológico sea entregado por el instructor como información, lo que no determina mayores diferencias entre la implementación presencial y la remota.

\section{CONCLUSIONES}

En general, creemos que este esquema mental de cuatro posibles causas puede ser una herramienta eficaz para enseñar a los estudiantes de medicina en formación a realizar una evaluación y manejo adecuado de un paciente con compromiso de conciencia.

Esta simulación puede llevarse a cabo de forma remota, ya que el objetivo está orientado a utilizar e integrar un esquema mental establecido para encontrar la causa del compromiso de conciencia y no para la práctica de habilidades clínicas.

\section{REFERENCIAS}

1. Forsberg S, Hojer J, Enander C, Ludwigs U. Coma and impaired consciousness in the emergency room: characteristics of poisoning versus other causes. Emerg Med J. 2009; 26 (2): 100-102.

2. Padilla-Zambrano HS, Ramos-Villegas Y, Manjarrez Sulbaran JJ. Coma y alteraciones del estado de conciencia: revisión y enfoque para el médico de urgencias. Rev. Chil. Neurocirugí. 2018; 44: 89-97. 
3. García S, Sauri Suárez S, Meza Dávalos E, Villagómez AJ. Estado de coma y trastornos de la conciencia: una revisión analítica desde un enfoque neurofuncional. Parte I. Rev Esp Med Quir. 2013; 18: 56-68.

4. P. de Castro. Paciente con alteración de conciencia en urgencias. Anales Sis San Navarra. 2008; 31 (suppl.1): 87-97.

5. Cooksley T, Rose S, Holland M. A systematic approach to the unconscious patient. Clin Med (Lond). 2018; 18 (1): 88-92.

6. Dalan R, Chin H, Hoe J, Chen A, Tan H, Boehm BO, Chua KS, et al. Adipsic Diabetes Insipidus-The Challenging Combination of Polyuria and Adipsia: A Case Report and Review of Literature. Front Endocrinol (Lausanne). 2019; 10: 630. doi: 10.3389/ fendo.2019.00630. PMID: 31620086; PMCID: PMC6759785.

7. Rodriguez A, Fogelfeld L, Robertson G. Hypernatremic Hydrophobic Transient Adipsia Without Organic or Severe Psychiatric Disorder. J Clin Endocrinol Metab. 2019; 104 (11): 5427-5430. doi: 10.1210/jc.201901446. PMID: 31373657.

8. Hiyama TY, Utsunomiya AN, Matsumoto M, Fujikawa A, Lin CH, Hara K, et al. Adipsic hypernatremia without hypothalamic lesions accompanied by autoantibodies to subfornical organ. Brain Pathol. 2017; 27 (3): 323331. doi: 10.1111/bpa.12409. Epub 2016 Aug 2. PMID: 27338632.

9. Croskerry P, Petrie DA, Reilly JB, Tait G. Deciding about fast and slow decisions. Acad Med. 2014; 89 (2): 197-200. doi: 10.1097/ACM.0000000000000121. PMID: 24362398.

10. Croskerry P, Singhal G, Mamede S. Cognitive debiasing 1: origins of bias and theory of debiasing. BMJ Qual Saf. 2013; 22 Suppl 2(Suppl 2):ii58-ii64. doi: 10.1136/ bmjqs-2012-001712.

11. Ashokka B, Ong SY, Tay KH, Loh NHW, Gee CF, Samarasekera DD. Coordinated responses of academic medical centres to pandemics: Sustaining medical education during COVID-19. Med Teach. 2020; 42 (7): 762-771. doi: 10.1080/0142159X.2020.1757634.

12. He S, Lai D, Mott S, Little A, Grock A, Haas MRC, et al. Remote e-Work and Distance Learning for Academic Medicine: Best Practices and Opportunities for the Future. J Grad Med Educ. 2020; 12 (3): 256-263. doi: 10.4300/JGME-D-20-00242.1. PMID: 32595840; PMCID: PMC7301927.

Correspondencia:

Soledad Armijo-Rivera

E-mail:soledad.armijo@gmail.com 